\title{
TAGUNG
}

\section{Das Projekt „Konvergenz der Rechte“: Leitlinien und Grenzen einer Integration im europäischen Privatrecht}

\author{
Nóra Szabó*
}

Im Jahre 2009 wurde die Zusammenarbeit „Konvergenz der Rechte“ von Peter-Christian Müller-Graff und Christian Baldus, Liliane Nau, Olivier Cachard und Ludovic Bernardeau sowie von Peter Jung ins Leben gerufen. Die deutsch-französisch-schweizerische Kooperation zielt auf die sogenannte differenzierte Integration im europäischen Privatrecht.

Der differenzierten Integration kommt mit den Erweiterungen der Europäischen Union in den Jahren 2004 und 2007 sowie den damit zunehmenden kulturellen, politischen und rechtlichen Unterschieden zwischen den Mitgliedstaaten immer größere Bedeutung zu. Als differenzierte Integration wird eine Rechtsvereinheitlichung im Kreise einer Teilmenge der EU-Mitgliedstaaten bezeichnet, die sich bei der heute vorhandenen Heterogenität wesentlich leichter durchsetzen lässt als eine die gesamte Gemeinschaft erfassende Regelung. Betroffen sind hauptsächlich Bereiche des Familienrechts, des Erbrechts und des Sachenrechts, die sowohl für die Wissenschaft als auch für die Praxis von zentraler Bedeutung sind und bisher noch nicht vereinheitlicht wurden. Grund für die Verzögerung der Vereinheitlichung ist der Umstand, dass die genannten Felder von nationalen, regionalen und kulturellen Besonderheiten sehr stark geprägt sind. Deshalb werden die Mitgliedstaaten ihre materiell-rechtlichen Modelle und Systeme, die oft identitätsbildende Elemente darstellen, sowie die damit verbundenen gesetzgeberischen Kompetenzen nicht aufge-

\section{Der Bürger und die Konvergenz der Rechte}

Erste Tagung des Netzwerks Konvergenz der Rechte

Heidelberg, 5.-7. November 2009

\section{Eröffnung}

Christian BALDUS, Universität Heidelberg Peter-Christian MÜLLER-GRAFF, Universität Heidelberg

Olivier CACHARD, Université de Nancy II

Peter JUNG, Universität Basel

\section{Einführung}

Der Bürger und die europäischen Rechtsordnungen

Paul LAGARDE, Université de Paris I

\section{Der Status der Person}

Der Familienname

Véronique DAVID-BALESTRIERO, Université de Nancy II

René REPASI, Universität Heidelberg Vivianne GERALDES FERREIRA, Universidade di São Paulo/Universität Heidelberg

Verbindung - Aufösung von partnerschaftlichen Beziehungen

Jacqueline FLAUSS-DIEM, Université de Picardie

Françoise FURKEL, Universität des Saarlandes Zoltán CSEHI, Eötvös-Loránd-Universität Budapest

Das Familienvermögensrecht

Ehegüterrecht

Maître Hugues LETELLIER, Paris

Maître Edmond JACOBY, Forbach

Laura CAVANDOLI, Università di Parma

* Nóra Szabó, LL.M., Doktorandin am Institut für geschichtliche Rechtswissenschaft an der Ruprecht-KarlsUniversität Heidelberg. 
ben wollen. Im Gegensatz zu anderen Bereichen, in denen die Vergemeinschaftung deutlich weiter fortgeschritten ist - wie etwa im Wirtschafts-, Verbraucher- und im allgemeinen Obligationenrecht - werden die projektrelevanten Gebiete oft als domaine réservé der nationalen Rechtsordnungen bezeichnet und damit praktisch für Angleichungsversuche zum Tabu erklärt. Daher wird zunächst eine Integration in kleineren Kreisen angestrebt.

In dem Projekt geht es hauptsächlich um die im Einflussbereich des deutschen und des französischen Zivilgesetzbuchs stehenden Rechtssysteme, also denjenigen des Mittelmeerraums und von Teilen Ostmitteleuropas. In diesen historisch verwandten Rechtssystemen soll untersucht werden, ob sie bezüglich einiger projektrelevanter Gebiete so nahe beieinander stehen, dass eine vollständige Rechtsangleichung zwischen ihnen möglich wäre.

Ein vollständiger Konsens ist jedoch höchstwahrscheinlich nicht realisierbar, da die Traditionen in diesen Rechtsgebieten trotz der gemeinsamen römisch-rechtlichen Grundlagen sehr spezifisch geprägt sind. Jedoch verlangen die praktischen Bedürnnisse (wie Eheschließung, Tod oder auch Vermögenserwerb in verschiedenen Mitgliedstaaten) nach möglichst einheitlichen Regelungen auch in diesen klassischen materiell-rechtlichen Bereichen.

Im Rahmen des Projekts haben bereits zwei Tagungen stattgefunden: im November 2009 in Heidelberg zum Thema „Der Bürger und die Konvergenz der Rechte" sowie im November 2010 in Nancy mit dem Schwerpunkt „Das Unternehmen und die Konvergenz der Rechte“. Als Fortsetzung wird 2011 eine Konferenz in Basel über „Die Obligationen und die Konvergenz der Rechte“ folgen. Im vorliegenden Bericht werden die Problemkreise des ersten Symposiums zusammengefasst.

\section{Erbrecht}

Bertrand ANCEL, Université de Paris II Götz SCHULZE, Université de Lausanne Tomasz KRZYWICKI, Jagiellonen-Universität Krakau/Universität Heidelberg

Sachenrecht

Nathalie PIERRE, Université de Nancy II Jean-Michel GASSER, Université de Nancy II Markus ARTZ, Universität Bielefeld Marek PORZYCKI, Jagiellonen-Universität Krakau

\section{Zusammenfassung}

Der Bürger und das Gemeinschaftsrecht Ludovic BERNARDEAU, Université de Nancy II

Erste Tagung des Netzwerks: Der Bürger und die Konvergenz der Rechte

Im November 2009 wurde im Internationalen Wissenschaftsforum der Universität Heidelberg die erste Tagung des Projekts veranstaltet. Das dreitägige Symposium fand in deutscher und französischer Sprache statt. Es wurde mit der Begrüßung durch die Dekane der Juristischen Fakultäten Christian Baldus, Olivier Cachard und Peter Jung eröffnet. Anschließend stellte Peter-Christian MüllerGraff die oben genannten Zielsetzungen des Projekts vor. Ferner betonte er den funktionalen Ansatz als methodische Vorgehensweise bei der Rechtsvergleichung: Demnach habe man nicht von bestimmten Institutionen der verschiedenen Rechtsordnungen auszugehen, sondern die Interessen, Konflikte sowie sozialen Funktionen zu entdecken und erst dann festzustellen, ob man zu gemeinsamen Institutionen - als mögliche Antwort auf ein Phänomen - gelangen könne.

Nach dieser Einführung führte Paul Lagarde in den Schwerpunkt der aktuellen Tagung, der Bürger und die europäischen Rechte, ein. Er machte in diesem Zusammenhang darauf aufmerksam, dass zwar heute von einer ,Unionsbürgerschaft" die Rede sei, in Bezug auf die projektrelevanten Gebiete bestehe jedoch der Widerspruch, dass hier kein „Europarecht“, 
sondern nur „europäische Rechte“ vorhanden seien, die teilweise $\mathrm{zu}$ grundverschiedenen Lösungen führen könnten. Daher sei es Aufgabe des Symposiums, möglichst viele hinter dem einheitlichen Begriff der „Unionsbürgerschaft" steckende materiell-rechtliche Divergenzen aufzuzeigen. Daraufhin konstituierten sich Arbeitsgruppen zu den einzelnen Themen, um intensive Diskussionen zu ermöglichen.

Der zweite Tag begann mit Referaten zu einzelnen Fragen des Status der Person. Véronique David-Balestriero schilderte die französischen, René Repasi die deutschen und Vivianne Geraldes Ferreira die portugiesischen Grundzüge des Namensrechts. Während der Vorträge und der darauffolgenden Diskussion wurde das Grundproblem dieses Rechtsgebiets deutlich: Der Familienname habe mehrere miteinander konkurrierende Funktionen, nämlich eine soziale Zuordnungsfunktion, eine Individualisierungsfunktion, ferner aber auch kulturelle Dimensionen. Die Frage sei, welche Funktion von der jeweiligen Rechtsordnung in den Vordergrund gestellt werde, denn jedes Land habe eine eigene Interessengewichtung, die häufig durch kulturelle Umstände und Traditionen geprägt sei. Es zeige sich zwar generell die Tendenz, dass das Bestimmungsinteresse des Individuums gegenüber der Zuordnung zur Familie immer mehr Raum gewinne. Fraglich sei jedoch, ob es hier eine gemeinsame Tendenz (eventuell zur Entwicklung des Namensrechts in die Richtung des Persönlichkeitsrechts) gebe sowie ob dementsprechend die rechtlichen Regeln überzeugend seien und falls ja, welche Gesichtspunkte sie rechtfertigen würden.

Als nächster Problemkreis wurden das Eherecht und die diversen Formen der registrierten oder eben nicht registrierten nichtehelichen Beziehungen nach deutschem, französischem und ungarischem Familienrecht von Jacqueline Flauss-Diem, Françoise Furkel und Zoltán Csehi dargestellt. Im Zusammenhang mit der Eintragungsmöglichkeit homo- und heterosexueller Partnerschaften tauchten mehrere bedeutende Fragen auf. Einerseits wurde untersucht, wie man die verschiedenen Modelle der Lebensgemeinschaften auf einen gemeinsamen Nenner bringen könne, denn die nationalen Regelungen seien sehr vielfältig und oft selbst in den einzelnen Staaten umstritten; dies sei nicht zuletzt auch auf rechtspolitische Fragen zurückzuführen. Andererseits wurde erörtert, dass die meisten Mitgliedstaaten die Ehe - und damit die Folgen der Scheidung oder des Todes eines Ehegatten - ausschließlich für verschiedengeschlechtliche Paare vorbehielten, während im Falle der Auflösung der Lebensgemeinschaften viele Aspekte, wie Sorge- und Unterhaltsansprüche aber auch andere vermögensrechtliche Fragen, nicht hinreichend geklärt seien. Einige Teilnehmer hielten die Lösung des ungarischen Obersten Gerichtshofes für überzeugend, der die Lebensgemeinschaft als eine atypische Form der Gesellschaft bürgerlichen Rechts (sogenannte Gefühls- und Wirtschaftsgemeinschaft) behandelt. Vorteil an dieser Institution sei, dass sie den meisten Rechtsordnungen bekannt sei, was der Harmonisierung als gemeinsamer Grundlage dienen würde. Andererseits wurde jedoch eingewandt, dass das Institut der Gesellschaft bürgerlichen Rechts hauptsächlich auf wirtschaftliche Zwecke ziele und es keine sorge- oder gar erbrechtlichen Gesichtspunkte enthalte und damit eine Regelung der Lebensgemeinschaft im Rahmen dieses Instituts problematisch sei. Dennoch waren sich die Teilnehmer darüber einig, dass es vorstellbar sei, dass die nichteheliche Lebensgemeinschaft trotz ihrer Vielfältigkeit in den nationalen Rechtsordnungen leichter harmonisierbar sei als die Ehe.

Nachmittags wurden das französische und das deutsche Familienvermögensrecht von Hugues Letellier und Edmond Jacoby aus der Perspektive der Praxis dargestellt. In ihren Vorträgen haben die Referenten auf mehrere grundlegende Unterschiede aufmerksam gemacht. So sei beispielsweise bemerkenswert, dass bei Scheidungen als Vermögensbewertungsregel in Deutschland das sogenannte 
Nominalitätsprinzip (das heißt der Wert des Vermögens richtet sich nach dem Tag, an dem man geheiratet beziehungsweise den $\mathrm{Ge}$ genstand erworben hat), in Frankreich dagegen das sogenannte Liquiditätsprinzip (das heißt das Vermögen wird am Liquidationstag wieder bewertet) angewendet werde. Die praktische Folge dieser Tatsache sei, dass man in Frankreich mehr prozessiere. Dagegen bringe für die Praxis die Regelung der Unterhaltsansprüche nach deutschem Recht mehr Sicherheit mit sich als das französische System. Im Letztgenannten habe der Richter nämlich größere Entscheidungsspielräume sowohl in Bezug auf das Abweichen vom Ehevertrag als auch im Zusammenhang mit dem Zuspruch von Unterhaltsansprüchen. Schließlich referierte Laura Cavandoli über die Grundzüge des italienischen Ehegüterrechts. Sie wies auf die Reform von 1975 hin und betonte, dass diese sowohl für das EheschlieBungsrecht als auch für vermögensrechtliche Aspekte von grundlegender Bedeutung sei: Unter anderem sei durch sie festgelegt, dass die Ehegatten sowohl moralisch als auch rechtlich gleichgestellt sind. Außerdem sei die Möglichkeit der ehevertraglichen Vereinbarung von Gütertrennung eingeführt worden. Die Referentin betonte auch, dass in Italien die kirchliche Eheschließung standesamtlich anerkannt und damit der bürgerlichen Eheschließung gleichgestellt sei, was vielen anderen europäischen Rechtsordnungen fremd sei. Zur Auflösung des ehelichen Güterstands führte Cavandoli an, dass diese grundsätzlich mit der Auflösung der Ehe eintrete, Modifikationen seien jedoch möglich.

Der dritte Tag begann mit Referaten der Arbeitsgruppe zum Erbrecht. Zunächst trug Bertrand Ancel den neuen Vorschlag der Europäischen Kommission zur Erbrechtsverordnung vor. Er stellte die Grundsätze der Verordnung (die Regelungen des internationalen Privatrechts, das Prinzip des letzten gewöhnlichen Aufenthalts und die Handhabung der Teilung der beweglichen und unbeweglichen Sachen) dar. Danach sprach er konkreter über den Inhalt des Vorschlags. Erörtert wurde vor al- lem, dass dieser keine zur Orientierung erforderliche Definition des letzten gewöhnlichen Aufenthalts enthalte, dagegen aber die Möglichkeit der erbrechtlichen professio iuris und die ordre-public-Klausel kenne. Anschließend beschrieb Götz Schulze das deutsche materielle Erbrecht. Zunächst kam er auf die Grundprinzipien (Testierfreiheit, gesetzliche Erbfolge, Pflichtteilsrechte) zu sprechen, dann ging er zur Aufteilung des Erbrechts im Bürgerlichen Gesetzbuch und zu den Zusammenhängen mit dem Familienrecht über. Schließlich zeichnete er die Unterschiede (bezüglich der Stellung des Miterben, der Teilung des Erbes, der Verfügungen von Todes wegen und der Regelung der Pflichtteilsrechte) sowie die Gemeinsamkeiten (unter anderem Prinzip der Testierfreiheit, Bedingungen der Form und des Inhalts des Testaments, Regeln für die gesetzliche Erbfolge, Aufhebung des Fideikommisses, Pflichtteilsrecht und Erbschein) des deutschen und französischen Rechts auf. Als letzter Referent der Gruppe stellte Tomasz, Krzywicki das im Jahre 2009 neu kodifizierte polnische Erbrecht vor, das sowohl in seinen Grundprinzipien als auch bezüglich seiner Detailregeln viele Gemeinsamkeiten mit dem deutschen, aber auch mit dem französischen Recht aufweise. Nach der Darstellung der testamentarischen Erbfolge sprach er kurz die Regelung des polnischen internationalen Privatrechts an, wonach der maßgebliche Anknüpfungspunkt die Staatsangehörigkeit des Erblassers zum Zeitpunkt seines Todes sei. Daraufhin stellte sich in der Diskussion mit besonderem Gewicht die Frage, welche Rolle das internationale Privatrecht bei der Angleichung spielen könnte.

Als letztes Thema wurde das Sachenrecht im Rahmen der Tagung erörtert. Jean-Michel Gasser schilderte kurz die Grundprinzipien des französischen Rechts bezüglich des Begriffs und der Klassifikation der Sachen und stellte dann die Regelung des Mobiliar- und des Immobiliarsachenrechts ausführlich dar. Danach ging man zu Fragen der verschiedenen Formen des Miteigentums und der Gesamthand über: Nathalie Pierre referierte 
über die französische ,indivision“, Marek Porzycki über das Miteigentum nach Bruchteilen und zur gesamten Hand in Polen. Diese Formen wurden in der darauffolgenden Diskussion mit den im deutschen Recht miteinander rivalisierenden Instituten der Gesamthand und des Miteigentums (nach Bruchteilen) verglichen. Schließlich sprach Markus Artz über die Prinzipien des dinglichen Rechtsgeschäfts nach deutschem Recht, ferner stelle er das Trennungs- und Abstraktionsprinzip - als besonderes Modell des Eigentumserwerbs - dar. Letztgenanntes lenkte die Diskussion zu weiteren Systemen des Eigentumserwerbs, näm- lich zu den durch die französische Lösung geprägten Rechtssystemen, die das sogenannte Konsensual- oder Kausalprinzip anwenden, ferner zum österreichischen und ungarischen Traditionsprinzip. Dieser Punkt wurde als besonders empfindlich eingestuft. Er werde bezüglich der Integration noch viele Fragen aufwerfen.

Zum Schluss fasste Ludovic Bernardeau die Hauptergebnisse der Tagung zusammen und schloss in der Hoffnung einer weiterhin effektiven Zusammenarbeit das Symposium ab.

\section{Die Zukunft Europas}

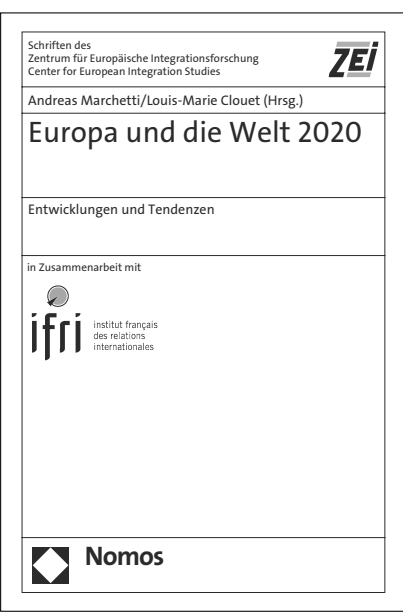

Bitte bestellen Sie im Buchhandel oder versandkostenfrei unter $\downarrow$ www.nomos-shop.de

\section{Europa und die Welt 2020}

Entwicklungen und Tendenzen

Herausgegeben von Andreas Marchetti und Louis-Marie Clouet

2011, ca. 220 S., geb., ca. 44,-€

ISBN 978-3-8329-6017-9

(Schriften des Zentrum für Europäische Integrationsforschung (ZEI), Bd. 74)

\section{Erscheint ca. Juni 2011}

Wie wird die EU 2020 aussehen? Welche Rolle wird sie international spielen? Welche Handlungsoptionen bieten sich ihr angesichts globaler Herausforderungen? Deutsche und französische Autoren zeigen auf, welche Entwicklungsmöglichkeiten sich mit Blick auf die kommenden Jahre bieten. Dabei wird deutlich: Welche Zukunft Europa haben wird, hängt maßgeblich von Europa selbst $a b$. 